\title{
NOTE ON A PAIR OF PROPERTIES WHICH CHARACTERIZE CONTINUOUS FUNCTIONS
}

BY C. H. ROWE

In a paper which appeared in this BuLleTıN* D. C. Gillespie discusses the class of functions of a single variable which share with continuous functions the property of never passing from one value to another without taking every intermediate value. He proves that a function of this class will be continuous if the set of values which it assumes an infinite number of times does not completely fill any interval, but he points out that this condition is not a necessary one. This leads us to ask whether we can form a condition for the continuity of a function of this class which is both necessary and sufficient, but which does not imply the continuity of a function which is otherwise unrestricted. An anwer to this question is given by the following theorem.

Necessary and sufficient conditions for the continuity of a function $f(x)$ defined in a closed interval $(a, b)$ are the following:

(A) If $x_{1}$ and $x_{2}$ are any two points of the interval, $f(x)$ takes each value between $f\left(x_{1}\right)$ and $f\left(x_{2}\right)$ in the interval $\left(x_{1}, x_{2}\right)$.

$(B)$ For every value of $\alpha$, the set $E(f=\alpha) \dagger$ is closed.

That these conditions are necessary is well known, $\ddagger$ and that $(B)$ alone does not imply continuity is shown by the example of any monotone discontinuous function, not constant in any interval. It thus remains to prove that the conditions are sufficient.

Let $\xi$ be any point in the interval and $\epsilon$ a given positive number. Denoting $f(\xi)$ by $\eta$, consider the sets $E(f=\eta+\epsilon)$

* Vol. 28 (1922), p. 245.

$\dagger$ This symbol denotes the set consisting of those points of the region in which the function is being considered at which the function is equal to $\alpha$.

$\ddagger$ See, e.g., W. H. Young, The Fundamental Theorems of the Differential Calculus, Cambridge Mathematical Tracts, No. 11, p. 7. 
[May-June,

and $E(f=\eta-\epsilon)$. Since each set is closed and since $\xi$ does not belong to either, there exists an interval $(\xi-\delta, \xi+\delta)$ which contains no point of either set and in which, therefore, $f(x)$ does not take either of the values $\eta+\epsilon, \eta-\epsilon$. It follows that in this interval $f(x)$ lies between $\eta+\epsilon$ and $\eta-\epsilon$, because if, for example, $f(x)$ took a value greater than $\eta+\epsilon$ at a point $\xi^{\prime}$ in this interval, it would take the value $\eta+\epsilon$ at some point between $\xi$ and $\xi^{\prime}$. Hence, $|f(x)-f(\xi)|<\epsilon$ if $|x-\xi|<\delta$.

An obvious modification of this proof will show that we may replace $(B)$ by the following less exacting condition which is similar in form to the condition given by Gillespie which we have quoted above:

The values of $\alpha$ for which $E(f=\alpha)$ is not closed do not completely fill any interval.

We may also replace $(B)$ by the following condition which is equivalent to $(B)$ whether $(A)$ is satisfied or not:

$(C)$ When $\alpha$ and $\beta$ are unequal, the distance between the sets $E(f=\alpha)$ and $E(f=\beta)$ is never zero.

By the distance between two sets is meant the lower bound of all possible distances from a point of one set to a point of the other. Hence, if $(C)$ is true, no limiting point of the set $E(f=\alpha)$ belongs to $E(f=\beta)$ unless $\beta$ is equal to $\alpha$, so that the set $E(f=\alpha)$ contains its limiting points and is closed. Conversely, the fact that two closed sets are at zero distance only when they have a common point* shows that $(B)$ implies $(C)$.

Analogous results hold for functions of several variables. We may state the following theorem, the proof of which presents no difficulties:

In order that a function $f(x, y, \cdot \cdot \cdot)$, defined in a finite open region $\dagger R$, may be continuous in $R$, it is necessary and

* See W. H. Young, Theory of Sets of Points, p. 175.

$\dagger$ A set of points is called an open region if each of its points is an internal point and if any two of its points can be joined by a polygonal line consisting of points of the set. A closed region is an open region together with its frontier points. 
sufficient that, as we move along any continuous curve (in Jordan's sense) whose points belong to $R$, the function should never pass from one value to another without taking every intermediate value, and that, for every value of $\alpha$, the set $E(f=\alpha)$ should contain those of its limiting points that belong to $R$.

It might seem that we should have had a more natural generalization of our original result if we had written "closed region" instead of "open region,"* but if we had done so, the theorem would have been false, for our conditions would not have been sufficient to ensure the continuity of the function at points on the frontier of the region. It is easy to construct an example which shows this.

Consider the two-dimensional closed region $\dagger$ consisting of those points whose rectangular coordinates $x, y$, satisfy the two inequalities $0<x \leqq 1,0 \leqq y \leqq 1+x+\sin (1 / x)$, together with the points on the line $x=0$ for which $0 \leqq y \leqq 2$. If at every point of this region we define a function which is equal to $x$ when $x>0$ and to $y$ when $x=0$, it will be seen that, although both our conditions are satisfied, this function is discontinuous at all the points on the line $x=0$ which belong to the region with the exception of the origin.

We can however secure the sufficiency of our conditions by imposing suitable restrictions on the character of the closed region in which the function is defined. For example, our theorem is valid if $R$ is a closed region satisfying the condition that, corresponding to any frontier point $P$ of $R$, there are values of $\delta$ as small as we please such that the points of $R$ whose distances from $P$ do not exceed $\delta$ form a set having the property that any two of its points can be joined by an arc of a continuous curve consisting of points of this set.

Trinity College,

DUBLIN

* If $R$ were a closed region, our second condition would be equivalent to saying that $E(f=\alpha)$ is closed.

$\dagger$ This region is practically equivalent to one discussed by W. H. Young (Theory of Sets of Points, p. 184), but its description occupies less space. 